\title{
Réseau des laboratoires de santé publique du Canada : soutien prioritaire pour les régions éloignées et isolées du Nord du Canada
}

\section{Groupe de travail sur les infections virales respiratoires ${ }^{1}$}

\begin{abstract}
Citation proposée : Groupe de travail sur les infections virales respiratoires. Réseau des laboratoires de santé publique du Canada : Soutien prioritaire pour les régions éloignées et isolées du Nord du Canada. Relevé des maladies transmissibles au Canada 2020;46(10):364-5. https://doi.org/10.14745/ccdr.v46i10a02f
\end{abstract}

Mots-clés : COVID-19, Canada, dépistage aux points de service

\section{Déclaration sur la distribution du matériel de dépistage aux points de service pour la COVID-19 - 26 août 2020}

Dans un esprit de vérité et de réconciliation et en vue d'assurer un accès équitable aux soins de santé, le Réseau des laboratoires de santé publique du Canada préconise la distribution prioritaire du matériel de dépistage aux points de service dans les communautés des régions éloignées et isolées du Nord. Ces communautés comptent des proportions beaucoup plus élevées de membres des Premières Nations, de Métis et d'Inuits que les communautés du Sud du Canada et sont souvent confrontées à des obstacles liés à l'accès rapide aux soins de santé conventionnels, comme les services de diagnostic. Les services ne sont pas toujours offerts sur place, et les difficultés liées au transport des échantillons peuvent retarder la réalisation des analyses, ainsi que le diagnostic et le traitement des patients. Ces lacunes peuvent par ailleurs entraver la recherche de contacts et la mise en œuvre de mesures de santé publique efficaces pour contenir les réseaux de transmission.

Il existe actuellement un nombre limité d'options de soins de proximité ou de points de service et de matériels disponibles pour le dépistage du SRAS-CoV-2, le virus qui cause la COVID-19. Le matériel et les fournitures de dépistage sont très limités, ce qui entraîne des retards dans les tests. Les données actuelles montrent que la plupart des transmissions se produisent au cours des cinq premiers jours de la maladie et, par conséquent, les retards dans les délais de diagnostic maximisent le potentiel de propagation du SRAS-CoV-2. Par conséquent, il est essentiel d'accorder la priorité à la distribution du matériel de dépistage du SRAS-CoV-2 aux points de service dans les régions où ils auront le plus grand impact sur la santé publique, y compris les communautés des régions éloignées et isolées du Nord partout au Canada.

La seule option pour les tests du SRAS-CoV-2 aux points de service, pour une utilisation hors d'un laboratoire actuellement
Cette oeuvre est mise à la disposition selon les termes de la licence internationale Creative Commons Attribution 4.0

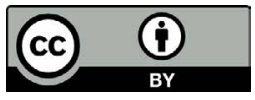

Affiliation

${ }^{1}$ Réseau des laboratoires de santé publique du Canada, Agence de la santé publique du Canada

Correspondance :

dionne.marcino@canada.ca disponible au Canada, est le test Xpert ${ }^{\mathrm{MD}}$ Xpress SARS-CoV-2 de Cepheid qui fonctionne avec le système GeneXpert ${ }^{\mathrm{MD}}$. Le test Xpert Xpress SARS-CoV-2 offre un délai d'exécution rapide d'environ 50 minutes, ainsi qu'une option d'interruption précoce du test après 30 minutes pour les échantillons positifs. Comme le système GeneXpert est utilisé pour dépister de nombreuses infections courantes, plus de 200 appareils sont déjà distribués au Canada, principalement dans les centres urbains du sud. Les centres urbains ont également accès à des services de test centralisés en laboratoire, avec des tests commerciaux à haut débit ou élaborés en laboratoire.

Les services de test centralisés en laboratoire ne sont pas disponibles ou sont difficiles d'accès pour les communautés des régions éloignées et isolées du Nord. À ce jour, 61 appareils aux points de service et plus de 9100 tests ont été distribués aux communautés éloignées, et les quantités de tests demeurent limitées. Par conséquent, il est essentiel d'élaborer une approche coordonnée pour la distribution des tests, afin que les communautés des régions éloignées et isolées du Nord reçoivent un approvisionnement suffisant pour fournir un soutien de diagnostic adéquat.

Comme elles n'ont pas accès en temps opportun à des services de test centralisés en laboratoire, certaines communautés des régions éloignées et isolées du Nord ont des délais d'exécution pour les tests de dépistage aux points de service de SRAS-CoV-2 qui peuvent dépasser 7 à 10 jours. De plus, les facteurs de risque des cas graves de COVID-19 sont prévalents dans les communautés des régions éloignées et isolées du Nord, notamment le diabète et les maladies cardiaques. Par conséquent, on craint que la propagation de la COVID-19 au 
sein de ces communautés et entre elles n'exerce des pressions sur le système de santé existant. L'attribution des ressources limitées du Canada devrait être guidée par des principes éthiques. Dans ce cas, une répartition équitable et juste des ressources conformément au principe de justice est primordiale pour répondre aux besoins, aux vulnérabilités et aux inégalités en santé qui en découlent pour les personnes vivant dans les communautés des régions éloignées et isolées du Nord.

Le Réseau des laboratoires de santé publique du Canada est d'avis qu'un soutien particulier devrait être fourni aux communautés des régions éloignées et isolées du Nord. Les autorités sanitaires provinciales et territoriales qui ont accès à des options de tests de diagnostics conventionnels en laboratoire sont encouragées à donner la priorité à la fourniture de matériel de dépistage et d'équipements de soins de proximité et de points de service aux communautés des régions éloignées et isolées du Nord. Tout en reconnaissant que la situation de chaque administration est différente, le Réseau des laboratoires de santé publique du Canada recommande que les provinces adoptent une approche coordonnée pour déployer une proportion importante de leur approvisionnement en tests Xpert Xpress SARS-CoV-2 dans les communautés des régions éloignées et isolées du Nord des provinces et territoires.

\section{Points clés de la présente déclaration}

- L'approvisionnement en tests aux points de service de Xpert Xpress SARS-CoV-2 est extrêmement faible au Canada.

- La plupart des tests aux points de service de Xpert Xpress SARS-CoV-2 sont réalisés dans des centres urbains où il existe d'autres options de test.

- $\quad$ En raison de ces pénuries de fournitures, il faut parfois jusqu'à 7 à 10 jours pour recevoir le résultat d'un test de dépistage du SRAS-CoV-2 dans les communautés des régions éloignées et isolées du Nord.

- Les affections associées à une forme grave de COVID-19, comme le diabète et les maladies cardiaques, sont répandues dans les communautés des régions éloignées et isolées du Nord.

- Les communautés des régions éloignées et isolées du Nord vulnérables qui n'ont pas un accès équitable aux services de test conventionnels devraient avoir la priorité pour le matériel de test au point de service, là où ils auront le plus grand impact sur la santé publique.

- Dès maintenant, et jusqu'à ce qu'il y ait une augmentation du matériel disponible au Canada, les autorités sanitaires devraient envisager de redéployer une proportion importante des matériels de test Xpert Xpress SARS-CoV-2 dans les communautés des régions éloignées et isolées du Nord, afin de contenir les vagues actuelles et futures de la pandémie.

- $\quad$ Cette priorisation des tests aux points de service pour le SRAS-CoV-2 respecte le principe de justice, assurant ainsi une répartition équitable des ressources en fonction des besoins et favorisant le bien-être de ceux qui, autrement, ne disposent pas d'options équivalentes pour les services de diagnostic rapide. 